This item was submitted to Loughborough's Research Repository by the author.

Items in Figshare are protected by copyright, with all rights reserved, unless otherwise indicated.

\title{
Trajectory planning for communication relay unmanned aerial vehicles in urban dynamic environments
}

PLEASE CITE THE PUBLISHED VERSION

https://doi.org/10.1007/s10846-017-0484-y

\section{PUBLISHER}

(C) Springer

VERSION

AM (Accepted Manuscript)

\section{PUBLISHER STATEMENT}

This work is made available according to the conditions of the Creative Commons Attribution-NonCommercialNoDerivatives 4.0 International (CC BY-NC-ND 4.0) licence. Full details of this licence are available at: https://creativecommons.org/licenses/by-nc-nd/4.0/

\section{LICENCE}

CC BY-NC-ND 4.0

\section{REPOSITORY RECORD}

Ladosz, Pawel, Hyondong Oh, and Wen-Hua Chen. 2017. "Trajectory Planning for Communication Relay Unmanned Aerial Vehicles in Urban Dynamic Environments”. figshare. https://hdl.handle.net/2134/24018. 


\title{
Trajectory Planning for Communication Relay Unmanned Aerial Vehicles in Urban Dynamic Environments
}

\author{
Pawel Ladosz • Hyondong Oh • Wen-Hua \\ Chen
}

the date of receipt and acceptance should be inserted later

\begin{abstract}
This paper proposes an optimal positioning and trajectory planning algorithm for unmanned aerial vehicles (UAVs) to improve a communication quality of a team of ground mobile nodes (vehicles) in a complex urban environment. In particular, a nonlinear model predictive control (NMPC)-based approach is proposed to find an efficient trajectory for UAVs with a discrete genetic algorithm while considering the dynamic constraints of fixed-wing UAVs. The advantages of using the proposed NMPC approach and the communication performance metrics are investigated through a number of scenarios with different horizon steps in the NMPC framework, the number of UAVs used, and heading rates and speeds.
\end{abstract}

\section{Introduction}

Ensuring communication amongst a group of mobile ground agents in a terrain with various obstacles is a difficult task due to obstructions and occlusions of signals, the restricted bandwidth and the limited communication range. In particular, in urban environments, numerous buildings cause line-of-sight (LOS) obstructions between mobile agents which may degrade communication signal strength significantly [1]. As a consequence, a ground-based radio can only be used over relatively short distances, rendering this type of solution inefficient and even infeasible. Satellite communication (SATCOM) is one of the alternatives to ground-based radios, however, it has limited availability as satellites follow pre-planned orbits with a certain spatiotemporal resolution and they can also be obstructed by buildings and other objects. So, this study proposes the use of small, low-flying and low power

This work was supported by the UK Engineering and Physical Science Research Council (EPSRC) under the Grant EP/J011525/1 and the Research Fund (Project Number 1.160089) of UNIST (Ulsan National Institute of Science and Technology).

Pawel Ladosz · Wen-Hua Chen

Department of Aeronautical and Automotive Engineering, Loughborough University, Loughborough, Leicestershire, LE11 3TU, United Kingdom E-mail: p.ladosz,w.chen@lboro.ac.uk Hyondong $\mathrm{Oh}$ (correspondence author)

School of Mechanical and Nuclear Engineering, Ulsan National Institute of Science and Technology (UNIST), Ulsan, Republic of Korea E-mail: h.oh@unist.ac.kr 
(in terms of communication) UAVs as urban communication relays. UAVs are able to solve most of the aforementioned shortcomings of SATCOM and ground-based radios using their mobility as they can: i) reach a mission area quickly without being confined to the ground road or limited availability and ii) rapidly change their position to mitigate a LOS obstruction problem once ground nodes change their position. Besides, the use of multiple smaller UAVs instead of a single larger one increases robustness of the network in case of partial platform (i.e. UAV or communication equipment) failures.

In order to determine the effective trajectory for UAVs to perform a communication relay mission, it requires the information on the ground node motion/behaviour. The ground node motion can be categorised into two scenario types: i) static, where ground nodes remain at a fixed position (or, at least, time needed for a change of position of ground nodes is much longer than that of UAVs) and ii) dynamic, where ground nodes change their position as the mission progresses. Both scenario types were explored in literature previously.

Static scenarios were widely covered in the literature. The algorithm described in [2] used UAVs to increase the second smallest eigenvalue of Laplacian matrix of the network graph leading to improved connectivity in a wireless sensor network. De Freitas et al. [3] showed an approach where the UAV is attracted to the last known neighbour, if the number of connection drops to one or less. Another approach was shown in [4] where a gradient following controller for a rotary wing UAV was developed. The algorithm was used to find the UAV position which improves all connections among a group of ground nodes. Dixon et al. [5] used a measured SNR and a gradient following technique to guide multiple UAVs to optimal positions between two ground nodes. Ho et al. [6] used the particle swarm optimisation (PSO) method to guide the UAV to the energy minimising path while maximising the connection time between the UAV and ground nodes. A data ferrying concept was explored by [7].

The majority of algorithms for static scenarios are not well-suited to mobile nodes as they do not consider dynamic constraints of UAVs. If the ground nodes change their positions, then a corresponding efficient communication structure can change quickly compared to time it takes for the fixed-wing UAV to react to that situation, particularly in an urban environment where obstacles (e.g. buildings) often cause a significant change in the communication performance. Thus, the need for different algorithms for mobile scenario arises. Mobile scenarios remains largely unexplored area and a few approaches were considered in the literature so far. Basu et al. [8] combined the number of connections to each UAV with a flocking algorithm to maximise the number of connected nodes at any given time. Approaches from $[9,10]$ tested several communication metrics to find the best position or trajectory for a team of UAVs. Dubins path and a simple communication disc model (which is based on a maximum communication radius) was used by [11] to find trajectories for a group of relay UAVs between a mobile ground node and a ground control station. Choi et al. [12] used UAVs to manoeuvre to minimise the ergodic link capacity amongst the UAV and a group of moving ground nodes. LOS obstruction by buildings in the urban environment has been rarely considered in the area of autonomous path/trajectory planning [13]. It is worthwhile noting that the presence of LOS obstructions introduces discontinuities and non-linearities into the communication quality between nodes as they cause significant signal quality reduction. 
This paper presents an optimal trajectory planning algorithm for communication relay UAVs to address the problem of mobile ground nodes in an urban environment. The new algorithm is built upon our previous approach [14]. The algorithm used in that paper was intended for static scenarios with the rotary-wing UAV (e.g. multi-rotors or helicopter type). Although the rotary-wing aircraft have a capability of being able to to hover around or over a single spot, they generally suffer from short endurances and low maximum speed. To mitigate these issues, fixed-wing UAVs are considered in this work. The algorithm proposed here is designed to: i) maximise communication performance with consideration of signal quality reduction due to obstacles (i.e. LOS obstruction), ii) obey dynamic constraints of fixed-wing UAVs and iii) be run as fast as possible for the online implementation.

To fulfil first two requirements, the nonlinear model predictive control (NMPC) trajectory planning algorithm proposed in [9] is adopted. The third condition is fulfilled by simplifying the algorithm by assuming a constant speed and discrete heading change of the UAV. The NMPC framework was chosen since the kinematic constraints of the UAV and obstacles can be easily incorporated into the problem formulation, while being adjustable to allow the real time implementation. Besides, as the NMPC updates the trajectory periodically, it can account for future deviations of initially predicted trajectories of ground nodes. To quantify the communication performance, this study uses the same communication model from [10] with three different communication performance metrics: the global message connectivity (GMC), the worst case connectivity (WCC) and the modified global message connectivity (mGMC). The GMC is used to improve the overall communication performance of the entire team while the WCC focuses the poorest/weakest link only. Meanwhile, the proposed mGMC uses a certain number of weak connections based on the desired communication quality requirement by combining the benefit of the GMC and the WCC.

The contributions of this paper can be summarised as: i) the urban environment with a large number of buildings which may obstruct the line-of-sight between nodes is explicitly considered in the communication cost; ii) the new communication performance metric, mGMC, is proposed, which can meet the communication requirement set by users; iii) the effective motion strategy for multiple relay UAVs is proposed to maximise the communication performance of the entire team using the proposed metric; and iv) the NMPC framework is used to address the underactuated fixed-wing UAV having dynamic constraints.

The paper is structured as follows. Section 2 describes a problem scenario and the overview of a trajectory planning algorithm. Section 3 provides details of a communication model and performance metrics. Section 4 discusses the proposed trajectory planning algorithm in detail. Section 5 provides numerical simulation results. Finally, section 6 provides conclusions and future work.

\section{Scenarios and Algorithm Overview}

Figure 1 shows a sample scenario considered for trajectory planning in this study. The yellow circles represent ground nodes/vehicles which are assumed to be performing their own individual missions, black dotted lines represent the trace of 
ground vehicles, aircraft models represent UAVs and blue cuboids represent buildings. Assumptions made in this work are listed as:

i) The urban environment is known prior to the mission;

ii) The UAVs can obtain the current position of the ground vehicles via communication; and

iii) If a line-of-sight (LOS) is obstructed by buildings between two nodes, a communication quality is reduced proportionally to the length of the obstruction.

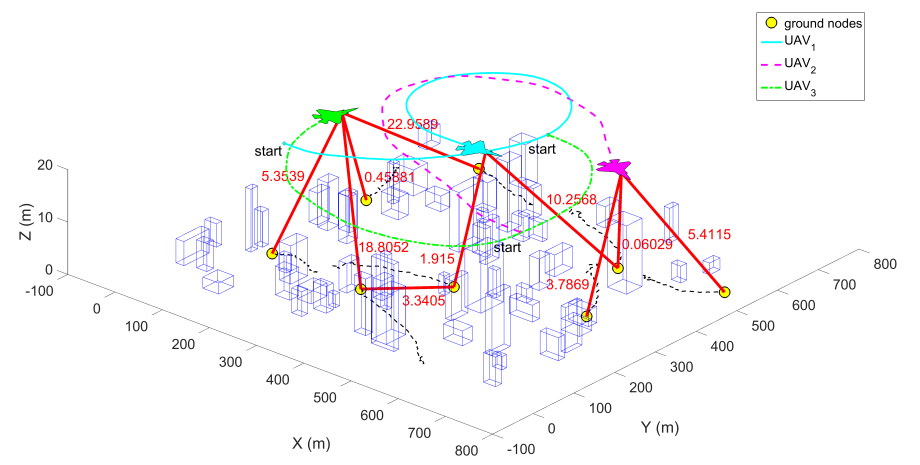

Figure 1: A sample scenario of the proposed approach.

The flow chart of the proposed trajectory planning algorithm for communication relay UAVs in an urban environment is shown in Fig. 2. Firstly, each UAV estimates states and also predicts the future position of ground vehicles for a certain time steps ahead with the Kalman filter and the GPS position of ground vehicles as sensor measurements. Based on position estimates, UAVs then calculate the communication cost in consideration of the communication range, signal-to-noise ratio and line-of-sight obstruction. The trajectory and the minimum spanning tree (MST) for a given UAV is found using a discrete genetic algorithm (GA)-based optimisation with the desired communication performance metric.

\section{Communication Modelling and Performance Metrics}

\subsection{Communication Model}

\subsubsection{Communication Probability}

Finding an optimal position requires establishing a node-to-node communication quality. In this work, a communication quality is represented as a probability of successful communication using an open space communication model $[9,10]$. In this model, node $i$ has the transmission power of $P_{i}$ and observes a noise $\sigma_{i}^{2}$ while 


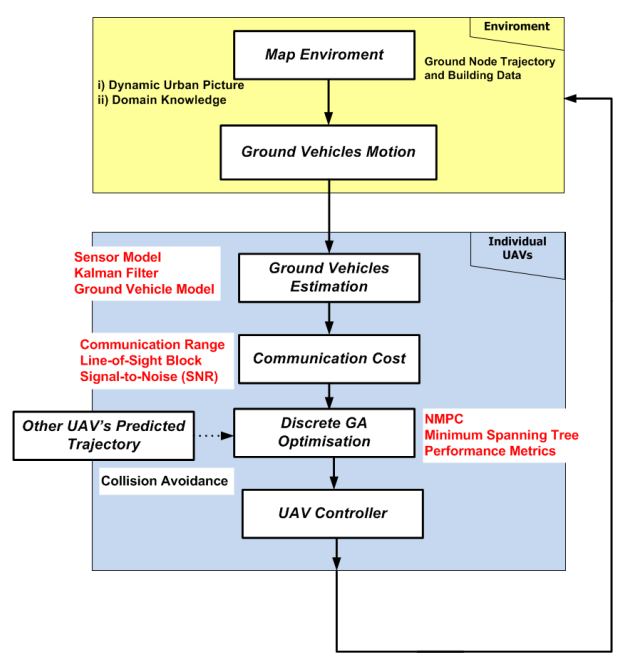

Figure 2: Flow chart of the NMPC-based trajectory planning algorithm for communication relay UAV.

receiving a signal from other members of the group. Then, the received signal-tonoise ratio (SNR) $\Gamma_{i j}$ for a signal transmitted from the $i$-th node and received by $j$-th node is given as:

$$
\Gamma_{i j}=\frac{P_{i} G_{i j}}{\sigma_{i}^{2}}
$$

where $G_{i j}$ is a channel gain and can be expressed as:

$$
G_{i j}=\frac{C_{i j}\left|h_{i j}\right|^{2}}{D_{i j}^{\alpha}}
$$

where $C_{i j}$ is a constant accounting for antenna gains and shadowing, $h_{i j}$ is responsible for multipath fading, $\alpha$ is a path loss factor and $D_{i j}$ is the distance between two nodes.

For the computation of a probability of successful communication, a minimal acceptable link quality $\gamma$ needs to be determined. If Rayleigh fading is assumed i.e. $h_{i j}$ is the complex Gaussian with zero mean and unit variance, then the probability of successful transmission from $i$-th node to $j$-th node is equal to:

$$
P_{r}^{i j}\left(\Gamma_{i j} \geq \gamma\right)=\exp \left(-\frac{\sigma_{j}^{2} \gamma D_{i j}^{\alpha}}{C_{i j} P_{i}}\right)
$$

It is worthwhile noting that, at this time, the model does not consider effects like diffractions and reflections of wireless signal, as this would significantly increase the computational load.

\subsubsection{Communication Cost}

For optimisation purposes, a probability calculated in Eq. (3) is transformed into a cost. Then, effects of LOS obstructions are added. The weighted edge cost between 
two nodes (i.e. $i$ and $j$ ) is defined by using the above probability of successful transmission as:

$$
W_{i j}^{o}=-\ln \left(P_{r}^{i j}\right)
$$

Above equation implies that the higher the probability of successful communication is, the lower the cost is, and this weight will be used as a cost to be minimised. In order to consider buildings, an additional weight is added whenever a connection passes through the building. This weight has a relatively high value to strongly discourage connections through buildings and is represented as:

$$
W_{i j}^{b}= \begin{cases}W_{i j}^{p b} & \text { if the LOS is blocked } \\ 0, & \text { otherwise }\end{cases}
$$

where $W_{i j}^{p b}$ is a penalty cost due to LOS block. The computation of LOS block is explained in the next section. Note that, non-smooth characteristic of $W_{i j}^{p b}$ can make the solution space highly non-convex with many local minima, as illustrated in Fig. 3(a). This figure shows a certain communication performance metric when using a single relay UAV at different grid locations at a constant altitude (different communication performance metrics will be explained in Section 3.1.3). To mitigate the issue, a new weight is introduced by using the length of LOS block $l_{b}$ inside the building (i.e. the length of the intersection/overlap between the LOS and the obstructing building as illustrated in Fig. 4) as:

$$
W_{i j}^{b}= \begin{cases}W_{i j}^{p b} l_{b} & \text { if the LOS is blocked } \\ 0, & \text { otherwise }\end{cases}
$$

where $l_{b}$ is the distance of LOS intersection with an obstructing building and $W_{i j}^{p b}$ is a base cost. Figure 3(b) clearly shows a much smoother cost function, which facilitates an optimisation process. Matlab library written in IRNA [15] is used to find the intersection and he distance $l_{b}$ between the LOS line and the building.

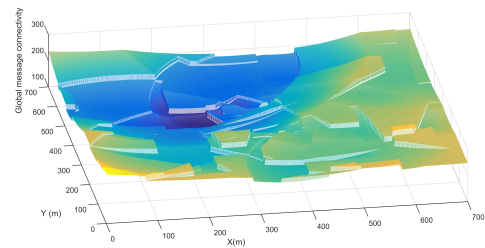

(a) Non-smooth weight due to discrete LOS obstruction.

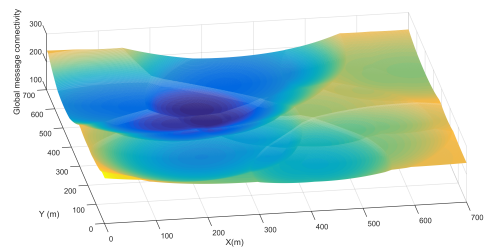

(b) New weight considering the length of overlap.

Figure 3: Surface plot for the communication performance function at different relay UAV locations.

For static scenarios, the weight for the UAV height restriction is also added. As in Eq. (3), a communication quality is better if nodes are close to each other, implying it is likely that UAVs' optimal position would be dangerously close to the 

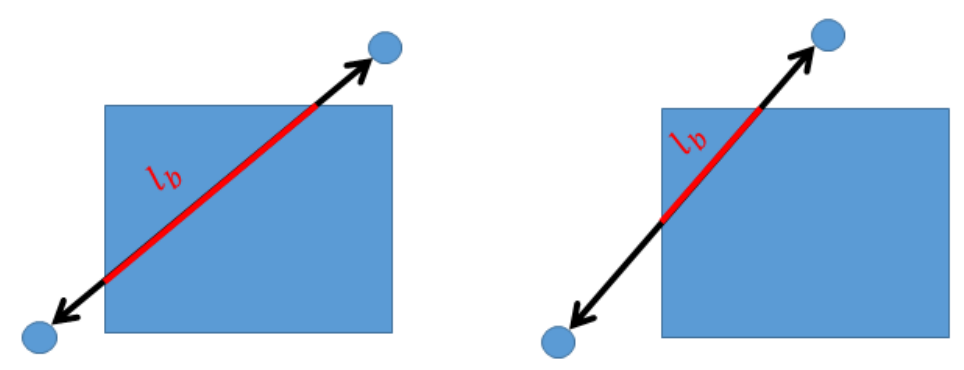

Figure 4: Illustration of new weight computation. The case on the left would result in a higher cost than that on the right since the longer portion of the LOS line overlaps with the building.

ground and buildings in order to maximise the communication quality. To prevent this from happening, a minimum allowable UAV height is imposed as:

$$
W_{i j}^{h}= \begin{cases}W_{i j}^{p h}, & \text { if below minimum height } \\ 0, & \text { otherwise }\end{cases}
$$

where $W_{i j}^{p h}$ is a penalty cost due to the height restriction. It is worthwhile noting that this weight is not related to the communication in a strict sense; it only serves as a barrier to prevent UAVs from flying too low.

Finally, the total communication weight, $W_{i j}^{t}$, can then be expressed as:

$$
W_{i j}^{t}=W_{i j}^{o}+W_{i j}^{b}+W_{i j}^{h} .
$$

As the number of nodes involved in a scenario increases, the number of connections in the network increases significantly. As a result, efficient sharing of information becomes a problem. To deal with this issue, this work uses a minimum spanning tree (MST) concept. The MST is defined as a subset of graph where all nodes are connected to each other but there are no loops, having a minimum (or at least the same as minimum, as there can be several minimum spanning trees in a single scenario) sum of edge weights [16]. In simple terms, the MST finds the least costly and least number of connections to connect all the members in the group.

\subsubsection{Communication Performance Metrics}

In the team of networked nodes, optimal UAV position or trajectory will be different depending on performance metric (index) used in optimisation. This work considers three communication metrics: the global message connectivity (GMC), the worst case connectivity (WCC), and the modified global message connectivity (mGMC).

Global message connectivity (GMC) The GMC is defined as a probability of message being successfully transmitted to all nodes within the minimum spanning tree (MST) [10]. As all positions of ground nodes, UAVs and buildings are known, Eq. (8) can be used to find node to node communication cost. With those individual link weight, the MST can be constructed. Let the Adjacency matrix of the 
MST be represented as $A^{\prime} \in \mathbb{R}^{(n+m) \times(n+m)}$, where $n$ is a UAV number and $m$ ground vehicle number, then $A_{i j}^{\prime}=1$ if the link from node $i$ to node $j$ is the part of the MST, and $A_{i j}^{\prime}=0$, otherwise. In the MST, a probability of message being successfully transmitted to all nodes is a sum of all connections within the MST, thus the performance index can be denoted as:

$$
J_{G M C}\left(\overline{\mathbf{x}}^{\text {pos }}, \overline{\mathbf{x}}^{g, p o s}\right)=\sum_{i=1}^{n+m} \sum_{j=1}^{n+m} A_{i j}^{\prime} W_{i j}^{t} .
$$

Note that $W^{t}$ and consequently $A^{\prime}$ depend on 3-D location of UAVs (represented as $\overline{\mathbf{x}}^{\text {pos }} \in \mathbb{R}^{3 \times n}$ ) and ground nodes (represented as $\overline{\mathbf{x}}^{g, \text { pos }} \in \mathbb{R}^{3 \times m}$ ). thus by minimising the GMC performance index $\left(J_{G M C}\left(\overline{\mathbf{x}}^{\text {pos }}, \overline{\mathbf{x}}^{g, p o s}\right)\right)$ with respect to UAV position, the communication relay optimal position can be found as:

$$
\min _{\overline{\mathbf{x}}^{\text {pos }}} J_{G M C}\left(\overline{\mathbf{x}}^{\text {pos }}, \overline{\mathbf{x}}^{g, p o s}\right)=\min _{\overline{\mathbf{x}}^{\text {pos }}} \sum_{i=1}^{n+m} \sum_{j=1}^{n+m} A_{i j}^{\prime} W_{i j}^{t} .
$$

A simple illustration of using the GMC as a performance index is shown in Fig. 5. This metric improves global communication quality rather than focusing only on a particular link(s).

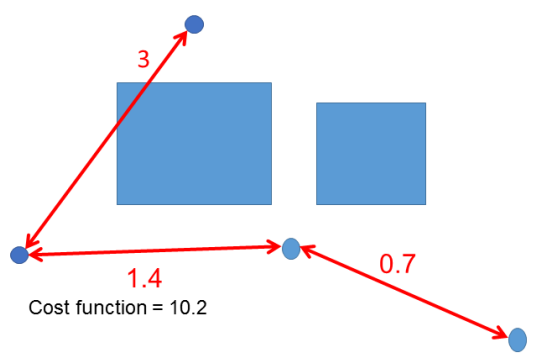

Figure 5: Cost function with the GMC explained on a simple example. The blue square is a building, blue circles are nodes and red lines is the MST.

Worst case connectivity (WCC) The WCC is defined as the link with the lowest probability of successful communication of all the links within the MST [10]. Using the MST definition from previous paragraph, UAV relay position can be found by minimising the weight of worst link within the MST with respect to UAV locations $\overline{\mathbf{x}}^{\text {pos }}$ as:

$$
\min _{\overline{\mathbf{x}}^{p o s}} J_{W C C}\left(\overline{\mathbf{x}}^{\text {pos }}, \overline{\mathbf{x}}^{g, p o s}\right)=\min _{\overline{\mathbf{x}}^{p o s}}\left(\max _{\forall i, j \in \mathbb{M}}\left(W_{i j}^{t} A_{i j}^{\prime}\right)\right)
$$

where $\mathbb{M}=\{1, \cdots, n+m\}$ is a set of all nodes in the network (i.e. $m$ UAVs and $n$ ground nodes). This performance metric is a worst connection within the MST as shown in fig. 6, and UAV is trying to improve that connection. This index performs exceptionally in case of numerous disconnected group of ground nodes. 


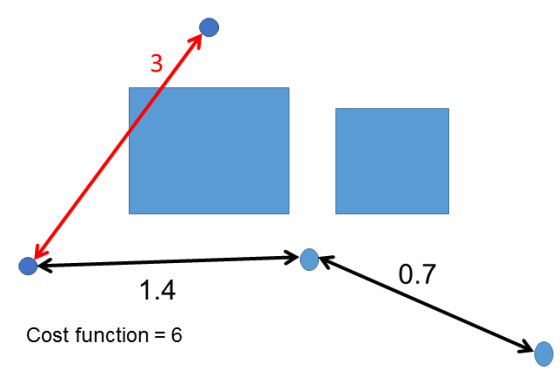

Figure 6: Cost function with the WCC explained on a simple example.

Modified global message connectivity $(m G M C)$ The aforementioned WCC and GMC focus on improving one worst connection and overall communication performance respectively. If a need arises to improve both overall and node to node communication quality, neither of the metric is suitable. To this end, the mGMC metric is proposed as a compromise between the GMC and the WCC. For the mGMC performance index, constant $\beta$ is defined as the number of weak connections in the initial MST to be improved. The constant can be obtained by considering user defined minimum communication probability $P_{d}$ of the network.

Firstly, desired probability needs to be converted into weight as:

$$
W^{d}=-\ln \left(P_{d}\right)
$$

then $W_{i j}^{t}$ for all $i, j \in \mathbb{M}$ is compared against $W^{d}$ to find connections weaker than the desired probability in the following manner:

$$
W_{i j}^{t c}= \begin{cases}1, & \text { if } W_{i j}^{t} A_{i j}^{\prime}>W^{d} \\ 0, & \text { otherwise }\end{cases}
$$

where $W^{t c} \in \mathbb{R}^{(n+m) \times(n+m)}$. Lastly, constant $\beta$ is calculated as:

$$
\beta=\sum_{i=1}^{n+m} \sum_{j=1}^{n+m} W_{i j}^{t c}
$$

$\beta$ is then used to compute the mGMC performance index, starting with rearranging the connectivity matrix into a vector:

$$
W^{t s}=\operatorname{vec}\left(W^{t} A^{\prime}\right)
$$

where $W^{t s} \in \mathbb{R}^{(n+m)^{2} \times 1}$. This vector is sorted in a descending order and stored as $W^{t s}$. The mGMC cost can then be found as:

$$
\min _{\overline{\mathbf{x}}^{p o s}} J_{m G M C}\left(\overline{\mathbf{x}}^{p o s}, \overline{\mathbf{x}}^{g, p o s}\right)=\min _{\overline{\mathbf{x}}^{p o s}} \sum_{k=1}^{\beta} W_{k}^{t s}
$$

The mGMC focuses neither on all connection within group nor the worst connections, but rather on improving number of connection decided by user requirements. Figure. 7 shows the computation using the mGMC metric on a simple example. For this case, as $\beta$ is 4 (considering bi-directional links) and $W^{d}=1$, the total mGMC metric cost is equal to $(3.0+1.4) \times 2=8.8$. 


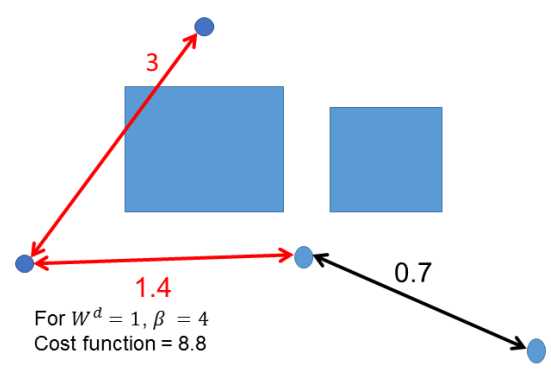

Figure 7: Cost function with the mGMC explained on a simple example.

\section{Relay UAV Optimal Positioning and Trajectory Planning}

Distinguishing and investigating the advantages of each performance metrics introduced from the previous Section can be performed easily on a static scenario as removing time dependence reduces the number of variables affecting the performance of each metric. Thus, static scenarios are considered as well. As the nature of the dynamic and static scenarios is different (i.e. the one requires trajectory planning with consideration of UAV dynamics while the other is simple positioning), each algorithm follows a different procedure to find an optimal solution.

\subsection{Relay UAV Optimal Positioning}

Firstly, in order to generate relay UAV optimal position for static scenarios using aforementioned performance metrics, the particle swarm optimisation (PSO) algorithm [17] is utilised. The PSO was used due to its ability to solve complex, non-smooth and non-convex problems. The PSO algorithm start by randomly spreading particles over the problem space and evaluating their cost function. The consecutive positions is found by computing velocity vector in each dimension of the problem as:

$$
v_{i, d}=r_{1} \omega v_{i-1, d}+\varepsilon r_{2}\left(X_{i b, i-1, d}-X_{c, i-1, d}\right)+\varepsilon r_{3}\left(X_{g b, i-1, d}-X_{c, i-1, d}\right)
$$

where $d$ denotes a problem space dimension, $i$ represents the iteration step, $\omega$ represents the inertia of the particle, and $\varepsilon$ is a correction factor. $X_{i b}, X_{g b}$ and $X_{c}$ represent the best position of a given particle, the global best position (considering all particles) and the current position of the particle, respectively. $v_{i, d}$ is the particle velocity, and $r_{1}, r_{2}, r_{3}$ are random numbers from zero to one. The calculation finish once convergence criterion (e.g. the maximum number of iterations) is satisfied.

\subsection{UAV Trajectory Planning}

In this section, trajectory planning for the fixed-wing communication relay UAV is discussed. The UAV trajectory planning problem in an urban environment presents several challenges. First of all, the fixed-wing type UAV (Fig. 8) considered in this 
paper is underactuated where the number of available control inputs is smaller than the number of controlled outputs. Besides, its control input (e.g. turning rates or speed command) is constrained and there is a nonholonomic (differential) state-vector constraint. These make it difficult for the fixed UAV to follow given arbitrary trajectories in 3-D space precisely. Moreover, due to the presence of buildings, the communication quality between nodes can change rapidly. For the implementation, the planning algorithm should be run in real time. Nonlinear model predictive control (NMPC)-based online trajectory planner has all of these features. UAV dynamic constraints can be added as optimisation constraints to be considered in the NMPC problem formulation. The algorithm can tackle rapid change of the communication quality. As the NMPC framework considers the future path of ground vehicles, the trajectory can also prevent the LOS obstruction from occurring in advance.

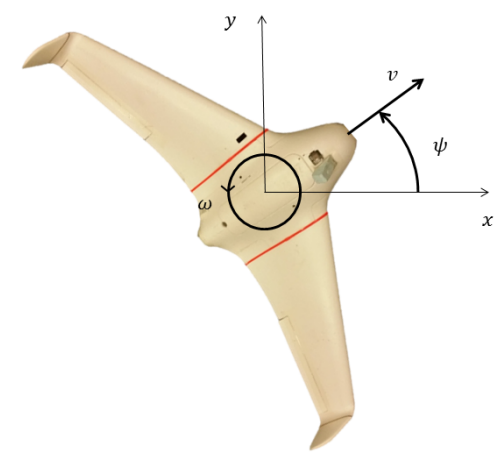

Figure 8: Example UAV with marked reference frame, speed, yaw rate and heading

\subsubsection{UAV Kinematic Model}

This work utilises a simple two-dimensional UAV kinematic model [18] by assuming the UAV speed is constant. This simplification was made to: i) reduce optimisation time and ii) save the energy used by the UAV during its flight, where the UAV model is given as:

$$
\left(\begin{array}{c}
\dot{x} \\
\dot{y} \\
\dot{\psi} \\
\dot{\omega}
\end{array}\right)=f\left(\mathbf{x}, u_{\omega}\right)=\left(\begin{array}{c}
v \cos \psi \\
v \sin \psi \\
\omega \\
-\frac{1}{\tau_{\omega}} \omega+\frac{1}{\tau_{\omega}} u_{\omega}
\end{array}\right)
$$

where $\mathbf{x}=(x y \psi \omega)^{T}$ are the inertial position, heading, speed and yaw rate of the UAV, respectively. $\tau_{\omega}$ is time constant accounting for actuator response delay, which can be determined experimentally for given UAV model. $u_{\omega}$ is a command input in form of turning rate. In this work, the command is constrained by:

$$
\left|u_{\omega}\right| \leq \omega_{\max }
$$




$$
\left|u_{\omega, k}-u_{\omega, k-1}\right|=0.1
$$

where $k$ is a current time step. The first constraint limits the maximum heading rate of the vehicle. The second constraint limits the rate at which heading changes and allows for discretisation of the controller. Both constraints are the result of dynamic limits of a fixed-wing UAV. The UAV model from Eq. (18) is discretised using Euler integration as:

$$
\mathbf{x}_{k+1}=f_{d}\left(\mathbf{x}_{\omega, k}, u_{k}\right)=\mathbf{x}_{k}+T_{s} f\left(\mathbf{x}_{k}, u_{\omega k}\right)
$$

where $\mathbf{x}_{k}=\left(\begin{array}{llll}x_{k} & y_{k} & \psi_{k} & \omega_{k}\end{array}\right)^{T}$ and $T_{s}$ is a sampling time.

\subsubsection{Tracking Filter with Ground Vehicle and Sensor Models}

For the UAV to predict future states of ground vehicles (position, velocity and acceleration) which is required for NMPC-based trajectory planning, the Kalman filter $(\mathrm{KF})$ is used. Generally, for a discrete $\mathrm{KF}$, two steps are required using vehicle and sensor models: i) prediction step where vehicle states and error covariance are extrapolated and ii) update step where a correction is made.

The ground vehicle model is based on the work from [19] where a discrete state

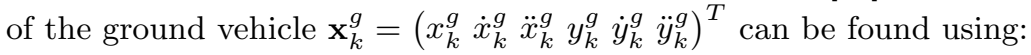

$$
x_{k+1}^{g}=F_{k} x_{k}^{g}+\eta_{k}
$$

where $\eta_{k}$ is a process noise which represents the acceleration characteristics of the target, and $F_{k}$ is a state transition matrix. The details of this model can be found in [19].

Provided that the ground vehicles are using GPS, their GPS positions are used as measurements for the UAV (via communication) for the position estimation as:

$$
\mathbf{z}_{k}=H_{k} \mathbf{x}_{k}^{g}+\mathbf{v}_{k}
$$

where the measurement matrix is:

$$
H_{k}=\left[\begin{array}{llllll}
1 & 0 & 0 & 0 & 0 & 0 \\
0 & 0 & 0 & 1 & 0 & 0
\end{array}\right]
$$

The measurement noise is $\mathbf{v}_{k} \sim N\left(0, R_{k}\right)$ and the covariance matrix is given as:

$$
R_{k}=\left[\begin{array}{cc}
\sigma_{x}^{2} & 0 \\
0 & \sigma_{y}^{2}
\end{array}\right]
$$

where $\sigma_{x}$ and $\sigma_{y}$ are the standard deviations of positions of $x$ and $y$, respectively.

\subsubsection{Introduction to NMPC-Based Trajectory Planning}

NMPC based trajectory planning algorithm is used to determine path for the UAV. To compute path NMPC combines finite time horizon with a non-linear model of the controlled system. Time horizon determines how far prediction of vehicle behaviour is made into the future. In order to account for behaviour throughout the prediction and not only at the beginning and end of prediction, the prediction time is discretised into $N$ horizon step, each of them of pre-specified length. At each horizon step change of control input to UAV is possible. Best possible combination of control inputs is then determined by optimisation procedure. At the end of optimisation, only control input from first horizon step is provided to actual controller, and procedure repeats for next time step. 


\subsubsection{Performance Index}

In a dynamic scenario, using performance metrics from Section 3.1.3 on its own is not sufficient as they do not account for dynamic properties of UAVs. Thus, the NMPC framework is combined with the aforementioned performance metric to create a trajectory plan for the UAV in the from of control input sequence $U^{i}=\left(u_{\omega, 0}^{i}, u_{\omega, 1}^{i}, \ldots, u_{\omega, N-1}^{i}\right)$ where $i$ is $i$-th UAV and $N$ is a horizon step. To find this control sequence, a new performance index is defined in Eq. (26) and minimised using a genetic algorithm (GA):

$$
J_{d}=\Phi\left(\bar{x}_{N}, \bar{x}_{N}^{g}\right)+\sum_{k=0}^{N-1} L\left(\bar{x}_{k}, \bar{x}_{k}^{g}, u_{\omega, k}^{i}\right)
$$

s.t.

$$
\begin{gathered}
\mathbf{x}_{k+1}^{i}=f_{d}\left(\mathbf{x}_{k}^{i}, u_{\omega, k}^{i}\right) \\
\omega_{\min } \leq u_{\omega, k}^{i} \leq \omega_{\max } \\
\left|u_{\omega, k}^{i}-u_{\omega, k-1}^{i}\right|=\Delta u_{\omega}
\end{gathered}
$$

where:

$$
\begin{gathered}
\Phi\left(\overline{\mathbf{x}}_{N}, \overline{\mathbf{x}}_{N}^{g}\right)=p_{c} J_{z}\left(\overline{\mathbf{x}}_{N}^{\text {pos }}, \overline{\mathbf{x}}_{N}^{g, p o s}\right) \\
L\left(\overline{\mathbf{x}}_{N}, \overline{\mathbf{x}}_{N}^{g}, u_{\omega, k}^{i}\right)=\frac{1}{2}\left[q_{c} J_{m G M C}\left(\overline{\mathbf{x}}_{k}^{\text {pos }}, \overline{\mathbf{x}}_{k}^{g, p o s}\right)+r_{v}\left(\frac{u_{\omega, k}^{i}}{\omega_{m a x}}\right)\right]^{2}
\end{gathered}
$$

where $J_{m G M C}$ represents the mGMC performance index and $\overline{\mathbf{x}}_{k}^{\text {pos }}$ and $\overline{\mathbf{x}}_{k}^{g, p o s}$ are $x$ and $y$ position of UAVs and ground vehicles, respectively, from state vectors $\mathbf{x}_{k}=$

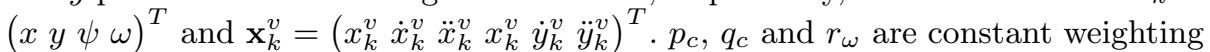
factors. It is worth noting that as the process is repeated at every sampling time, only first control input from $u_{\omega, k}$ is implemented to control corresponding UAV.

Due to the large computation time to obtain desired commands, a decentralised approach is used in this work. The decentralised approach implies that each UAV needs to determine the MST independently and calculate its optimal control based on future predictions of other UAVs and ground vehicles, meaning that communication between UAVs is required within one sampling time. The sampling time can be adjusted to match the capability of communication equipment on-board UAVs. If all UAVs have the same positional information about each other, then each MST would be the same. However, it is possible that due to disruption and delays, each UAV would have different MSTs. One possible way of mitigating the issue is for UAVs to share their MST, and choose one with the lowest cost. The decentralised approach is also more robust since if one UAV fails, others can continue a communication relay mission. 


\subsubsection{Discrete Genetic Algorithm}

Finding the optimal control sequence $U^{i}$ is a non-convex optimisation problem with many constraints. Additionally, the need for fast computation further restricts the choice of optimisation algorithms. Heuristic algorithms like genetic algorithm (GA) or simulated annealing might show a promise in fulfilling desired requirements. In particular, a genetic algorithm has been shown to efficiently plan a path for a UAV target tracking problem in [20] with discrete control; thus this work utilises a similar GA algorithm, version of which is more broadly described in [21]. At the initialisation stage of GA, a first generation is created. It is a very important part of every GA as it largely determines the quality of problem space exploration and thus the quality of an available solution. Each chromosome consists of potential control sequence $U_{p}^{i}$ and can be denoted as:

$$
U_{p, j}^{i}=\left(u_{\omega, 0}^{i} u_{\omega, 1}^{i} \ldots u_{\omega, N-1}^{i}\right) \quad \forall j \in 1,2 \ldots, M
$$

subject to

$$
u_{\omega, k+1}^{i}=u_{\omega, k}^{i}+a \Delta u_{\omega}
$$

where $M$ is a population size, $N$ is a horizon step number and $a$ is chosen randomly from the set $\{-1,0,1\}$. Initial member of chromosome group $g_{0}$ is based on the control command executed in the previous step. Each subsequent command in a given sequence is determined by Eq. (33).

Each chromosome needs to be evaluated to determine its quality. The solution fitness $f$ of each chromosome is based on the value of $J_{d}$ defined in Eq. (26). A solution fitness can be found as:

$$
f=\frac{1}{\left(J_{d}+J_{e}\right)^{b}}
$$

where $b$ is a factor determined experimentally to prevent one solution from overwhelming the optimisation process too early, and $J_{e}$ is an additional cost which can be defined as:

$$
J_{e}= \begin{cases}A, & \text { if Eq. }(28) \text { or }(29) \text { is not satisfied. } \\ 0, & \text { otherwise }\end{cases}
$$

where $A>0$ is a penalty value due to constraint break.

At the reproduction stage, a new population is created while taking into account for the fitness evaluation. The process has three steps: selection, crossover and mutation.

Selection In selection, a set of breeding population is chosen from the already existing population. Selection is based on a widely used roulette wheel method. Let us define a set of chromosomes as:

$$
C=\left\{U_{p, 1}^{i}, U_{p, 2}^{i}, \ldots, U_{p, M}^{i}\right\}
$$

and a set of fitness function corresponding to each chromosome as:

$$
F=\left\{f_{1}, f_{2}, \ldots, f_{M}\right\}
$$


The sum of a fitness functions is then calculated as:

$$
F_{s}=\sum_{i=1}^{M} f_{i}
$$

Also, a cumulative fitness function as a percentage of a total cost function is defined as:

$$
F c=\left\{f c_{1}, f c_{2}, \cdots, f c_{M}\right\}
$$

where

$$
f c_{j}=\sum_{i=1}^{j} \frac{f_{i}}{F_{s}} \times 100 ; \quad \forall j \in\{1,2, \cdots, M\}
$$

Then uniformly distributed random number $\mu \sim \mathcal{U}\{0,100\}$ is generated and compared against cumulative fitness function. Last parent for who cumulative percentage fitness is smaller than the random number is used in a breeding population. This can be formally expressed as:

$$
G m_{k}=\underset{U_{p, j}^{i} \in C}{\arg \min }\left(f c_{j}-\mu \geq 0\right) ; \quad \forall k \in\{1,2, \cdots, M\}
$$

where $G_{m}$ is a chromosome from a breeding population. The process continuous until there are $M$ parents in a breeding population. Such a selection leaves the majority of the breeding population as good parents while allowing for space exploration by keeping a few bad ones.

Crossover Crossover is used to combine two parents chromosomes together which follows the procedure outlined below.

1. Two parents chromosomes $G m_{x}$ and $G m_{y}$ are randomly selected from a breading population.

2. Crossover point $g_{c}$ is randomly selected within two parents strings where $c \in$ $\{1,2, \cdots, N-1\}$ and crossover point is between $c$ and $c+1$

3. Parts from parent from start to crossover point and from crossover to to the end of other parent are added added.

Mutation To help with problem space exploration, mutation is used as a final step of reproduction. Mutation is calculated separately for each member of each chromosome. As this is very short optimisation and problem space needs to be explored quickly, for the majority of optimisation, mutation is set as a chance of $50 \%$ i.e. each member of each chromosome has a $50 \%$ chance of mutation. For the final few iterations, mutation is set for a significantly lower $17 \%$ chance to allow for convergence. If a given member mutates, then its new value can be denoted as follows:

$$
u_{\omega, k}^{i}=u_{\omega, k-1}^{i}+a \Delta u_{\omega}
$$

where $k$ is a mutating chromosome index and $a$ is a number randomly selected from set $\{-1,0,1\}$; although this might lead to a situation where constraints Eqs. (28) and (29) are violated, such solutions are quickly removed due to a high cost correlated with constrain violation.

Lastly, a convergence criterion for the GA algorithm is the number of acceptable generations $N_{g}$. 


\section{Numerical Simulations}

Static scenarios are first considered to perform initial comparison of three communication performance metrics: the global message connectivity (GMC), the worst case connectivity (WCC) and the modified global message connectivity (mGMC). After validating basic properties, dynamic scenarios are then considered to investigate the effect of moving ground vehicles on the proposed mGMC performance. Simulation parameters for communication equipment are based on a low-cost and low-power commercial off-the-shelf Ubiquity Pico station M2, as shown in Table 1.

Table 1: Radio communication parameter

\begin{tabular}{lll}
\hline Parameter & Value & Unit \\
\hline \hline Transmission power $\left(P_{i}\right)$ & 0.01 & $\mathrm{~W}$ \\
Noise power $\left(\sigma^{2}\right)$ & $1 \times 10^{-9}$ & $\mathrm{~W}$ \\
Max communication range & 300 & $\mathrm{~m}$ \\
Attenuation factor $(\alpha)$ & 3 & $\mathrm{n} / \mathrm{a}$ \\
Antenna gain $C$ for UGV and UAV & 1 & $\mathrm{n} / \mathrm{a}$ \\
\hline
\end{tabular}

\subsection{Static Scenarios}

\subsubsection{Sample Scenario}

To establish the basic behaviour of communication performance metrics, a sample scenario with two relay UAVs, 12 ground nodes and 30 buildings is discussed. Figure 9 shows the result of using different communication performance metrics in the optimisation process. For $J_{m G M C}$ cost computation, $W^{d}=20$ is used as a desired communication requirement, resulting in the number of weak connections $\beta=6$ in the for the static scenario unless otherwise stated.

Firstly, Fig. 9(a) presents MST between ground nodes (shown as red lines) without relay UAVs. Numerous connections are weak due to: i) buildings obstructing line-of-sight (LOS) and ii) relatively large distances between nodes. The weakest connection within the MST is between nodes 11 and 12 (shown as green line) with the weighted edge cost of $J_{W C C}=39.01$. The sum of all connection (termed as the global cost, hereafter) in the MST for this sample scenario is $J_{G M C}=281.4$, and the sum of $\beta$ worst connections is $J_{m G M C}=167.2$. Utilising the GMC as the performance metric in the optimisation process is shown in Fig. 9(b). As expected, using this metric reduces the global cost significantly to $J_{G M C}=154.12$. However, the connection between nodes 6 and 12 becomes very weak $\left(W_{612}^{t}=20.13\right)$ since the weak connection is not particularly considered; the purpose of the GMC metric is to improve all connection overall. Figure. 9(c) shows the result of using WCC as the performance metric in optimisation. This metric improves the value of worst connection to 16.3 between nodes 6 and 12. However, the global cost $J_{G M C}=186.04$ is the worst amongst three metrics. This comes from the 


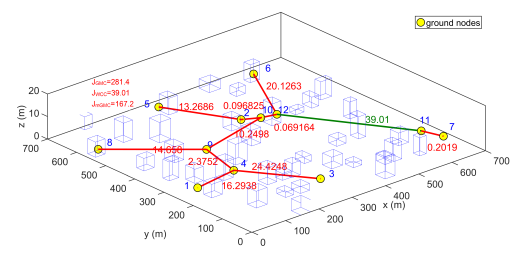

(a) MST without UAVs

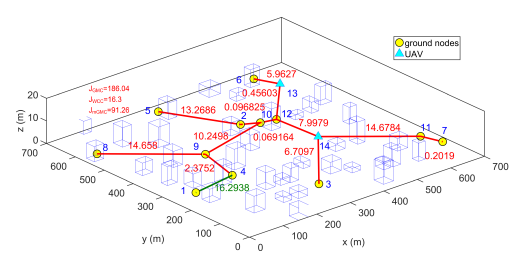

(c) Using the WCC with two UAVs

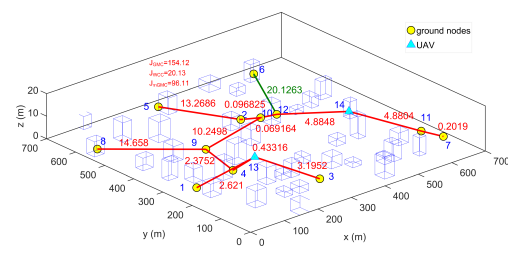

(b) Using the GMC with two UAVs

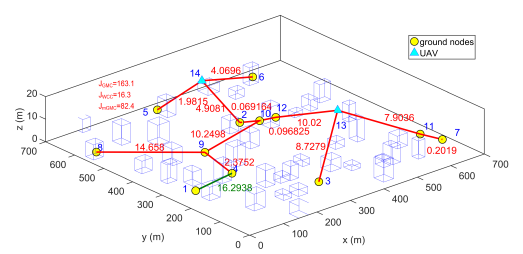

(d) Using the mGMC with two UAVs

Figure 9: Optimal deployment results for relay UAVs using different communication performance metrics.

nature of this metric which focuses on improving the weakest connection only while ignoring the global communication quality (i.e. all other connections).

Lastly, Fig. 9(d) shows the result of the proposed metric, mGMC. The resulting global cost for this metric is $J_{G M C}=163.1$ which is in the middle between the GMC and the WCC at 154.12 and 186.04, respectively. Besides, the worst connection cost $J_{W C C}=16.3$ is the same as using WCC as a metric. For the sum of three bi-directional worst connections (i.e. $\beta=6$ ), this metric provides the best result $\left(J_{m G M C}=82.4\right)$ among three metrics. As the worst connection value is below 20, using the proposed mGMC metric satisfies the desired communication requirement, while providing the reasonable global communication performance as well.

\subsubsection{Monte Carlo Simulations}

Monte Carlo simulations are used to confirm the aforementioned trends observed in the sample scenario. Figure 10 shows the results averaged over 30 independent Monte Carlo simulation runs. The global cost $\left(J_{G M C}\right)$ change with the increasing number of UAVs is shown in Fig. 10(a) using three different performance metrics. It is worthwhile noting that in this simulation, $W_{d}$ is adjusted so that $\beta=6$ at all times. As expected, the GMC metric (shown as a blue solid line) produces the best result, however the result from the mGMC is not much worse, which is particularly the case for the small number of UAVs. With the small number of UAVs, there are a large number of bad connections and thus improving them led to a significant improvement on the global cost using either of the GMC or the mGMC. Similar trend is shown in the WCC, however, the metric gets worse much faster than the mGMC. 
Figure. 10(b) with the WCC cost shows the reversed situation as in Fig. 10(a). This means that using WCC as the performance index provides the best result in terms of improving the worst connection, while the result from the GMC metric is the worst. Comparing three three metrics with the sum of $\beta=6$ worst connection cost $\left(J_{m G M C}\right)$ as shown in Fig. 10(c), it can be seen that the mGMC metric case shows the best performance. It can also be seen that adding more UAVs gets less improvements. This is because as UAVs are added into the scenario, the number of bad connections is reduced until there are only good ones to improve.

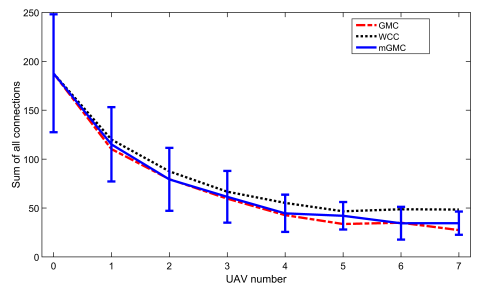

(a) $J_{G M C}$ cost

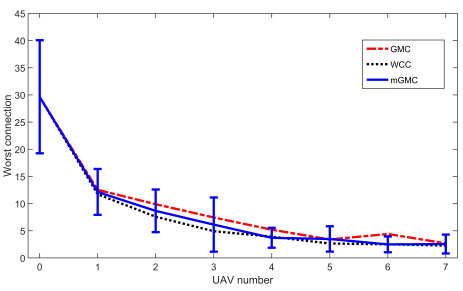

(b) $J_{W C C}$ cost

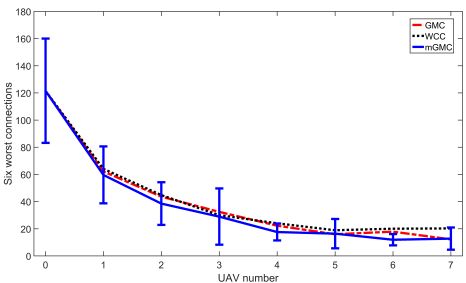

(c) $J_{m G M C}$ cost

Figure 10: Monte Carlo simulation results using three different communication performance metrics with different number of UAVs.

\subsection{Dynamic Scenarios}

Having shown comparison of the proposed mGMC metric with other metrics on static scenarios, investigation of this metric on dynamic scenarios is performed. It is worthwhile noting that due to movement of ground vehicles and corresponding communication quality change, $\beta$ also changes; thus, it is calculated at each simulation step to take into account all connections above $W^{d}$. The dynamic scenario with three UAVs, 12 ground nodes and 30 buildings uses the same communication parameters from Table 1, and other simulation parameters are shown in Table 2. In this section, the effect of following changes are mainly considered: i) change of horizon length in the NMPC problem formulation, ii) different movement pattern including random, loitering and the proposed method, iii) the number of UAV used, and iv) kinematic constraints of the UAV including the speed and turning rate. 
Table 2: Mobile simulation parameter

\begin{tabular}{lll}
\hline Parameter & Value & Unit \\
\hline \hline Actuator delay $\left(\tau_{\omega}\right)$ & $1 / 3$ & $\mathrm{~s}$ \\
UAV speed $(v)$ & 20 & $\mathrm{~m} / \mathrm{s}$ \\
Heading rate constraint $\left(\omega_{\min }, \omega_{\max }\right)$ & $(-0.2,0.2)$ & $\mathrm{rad} / \mathrm{s}$ \\
Receding horizon step $(N)$ & 5 & $\mathrm{~N} / \mathrm{A}$ \\
Maximum heading rate change $\left(\Delta U_{\omega}\right)$ & 0.1 & $\mathrm{rad} / \mathrm{s}$ \\
Weighting factors $\left(p_{c}, q_{c}, r_{\omega}\right)$ & $\left(1000, p_{c} / N, 1\right)$ & $\mathrm{N} / \mathrm{A}$ \\
Population size $(M)$ & 15 & $\mathrm{~N} / \mathrm{A}$ \\
Acceptable number of generations $\left(N_{g}\right)$ & 15 & $\mathrm{~N} / \mathrm{A}$ \\
\hline
\end{tabular}

Firstly, Fig. 12 shows that the horizon length has a critical impact on the performance when using the mGMC. If the horizon length is too small, UAVs will not see any possible trajectory which can improve the mGMC cost as the mGMC is only focused on a few worst connection, which might not be in that UAVs range. This situation is illustrated in Fig. 11. On the other hand, if the horizon is too long, ground vehicles' plan needs to be known well in advance and UAVs may overlook imminent weak connections for the sake of improving connections far away. To trade off between those two problems, the horizon length needs to be carefully determined. There might be two ways to change the horizon length: i) increasing the horizon steps with a fixed time interval or ii) increasing time interval between horizon steps with a fixed number of horizon steps. First method would result in significantly increased computational efforts but an accurate solution while the second does not increase computational efforts, but it reduces the accuracy of a solution. Since the computational speed is one of the requirements for the proposed algorithm as stated in the introduction, the second method is employed in this work. Table 3 shows the different time intervals between horizon steps with five horizon steps (which results in different total horizon length from 5 to 25) used in the numerical simulation shown in Fig. 12. It can be seen that there is a significant performance improvement up to the horizon length of 15 and smaller improvement between 15 and 20. After the horizon length of 20, the performance of the mGMC cost starts to decrease (so value increases) again and can be associated to UAVs trying to improve connections too far in the future. Thus, the best horizon length for the mGMC in this size of scenario is 20 and this horizon length will be used in other simulations hereafter.

Table 3: Different time intervals between horizon steps

\begin{tabular}{ll}
\hline Total horizon length $[\mathrm{sec}]$ & time interval between horizon steps [sec] \\
\hline \hline 5 & $(0.5,0.5,1,1,2)$ \\
10 & $(0.5,0.5,2,3,3)$ \\
15 & $(0.5,2.5,3,4,5)$ \\
20 & $(0.5,4.5,5,5)$ \\
25 & $(0.5,4.5,6,6,8)$ \\
\hline
\end{tabular}




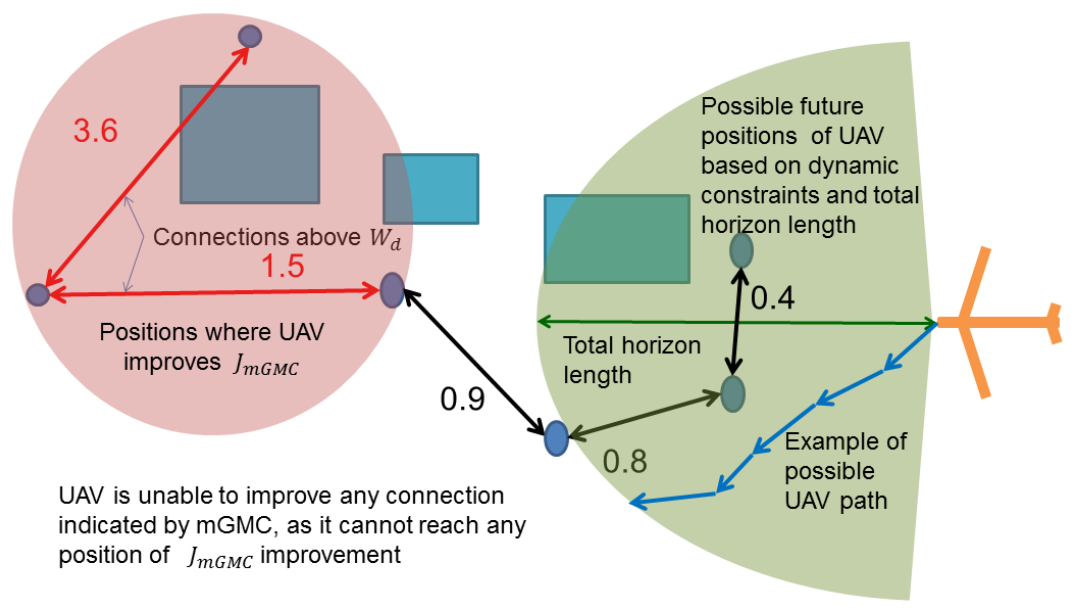

Figure 11: Illustration of a problem of too short horizon length where the UAV cannot see a move which would reduce the mGMC cost.

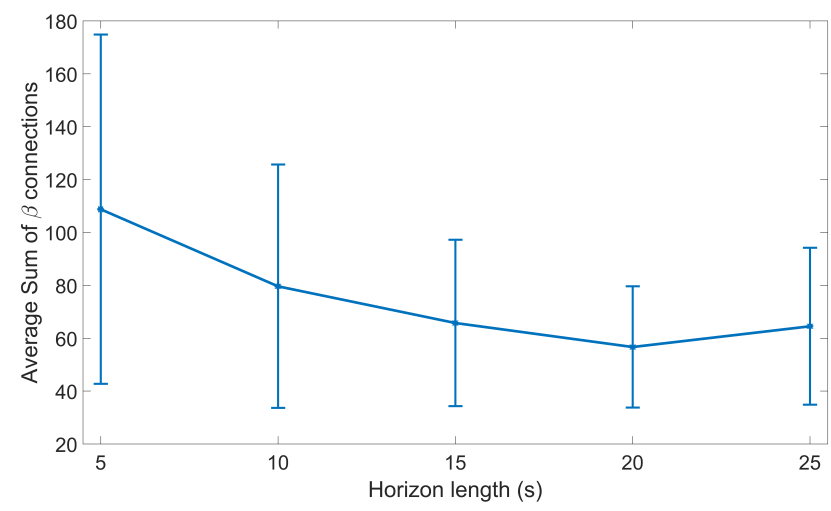

Figure 12: Averaged sum of $\beta$ connections over ten scenarios.

Figure 13 shows comparison between random movement, loitering (circling) around a starting point and using the proposed GA optimisation. At the beginning, all three methods show similar performance and the loiter shows even better performance during around 30 and 40 seconds of simulation. This might be because fixed-wing UAVs cannot change its heading or position instantaneously towards the optimal heading/position due to their dynamic constraints. However, the proposed method outperforms the other two for most of the time as it optimised its movement considering the movement of all ground nodes and the corresponding mGMC cost.

The effect of changing the number of UAVs is shown in Fig. 14. As expected, the more UAVs are used, the better the sum of $\beta$ connections is obtained. Note that the difference between two and three UAVs is not that significant. This implies that there is a saturation point where adding more UAVs does not result in a significant increase of communication performance. As more UAVs are added, there are fewer 


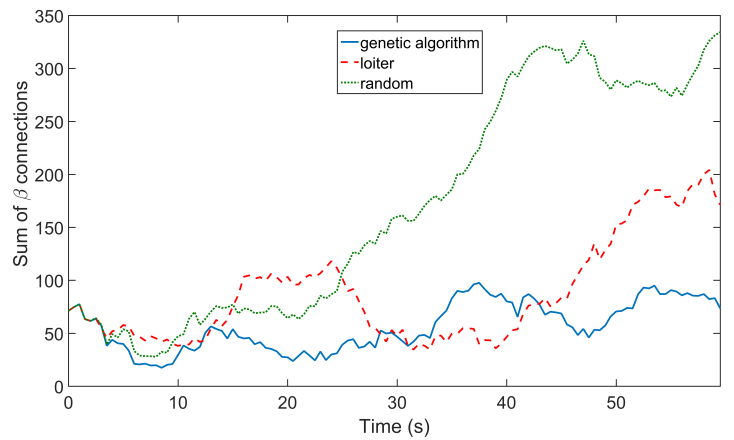

Figure 13: Comparison of NMPC-based trajectory planner using the genetic algorithm implementation with random motion and loitering.

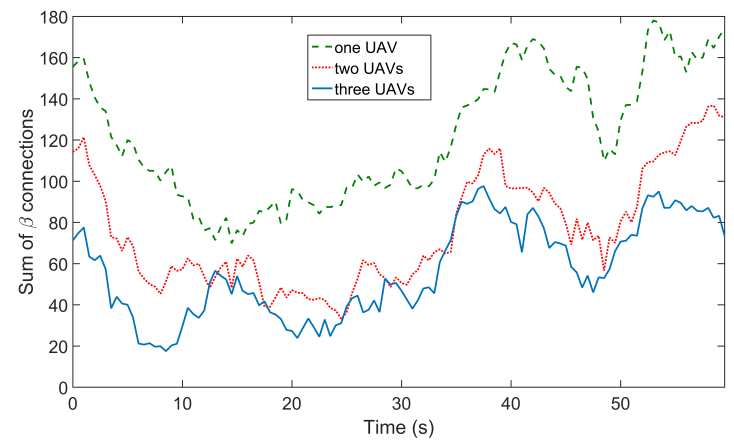

Figure 14: Effect of changing number of UAVs on the performance of the mGMC metric.

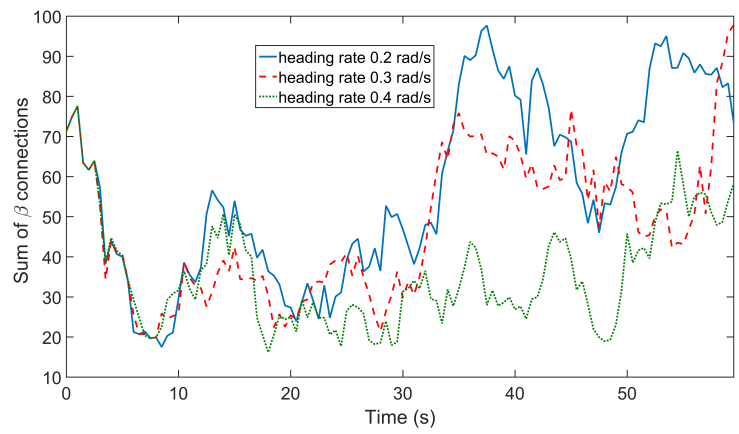

Figure 15: Effect of changing heading rate on the performance of the mGMC.

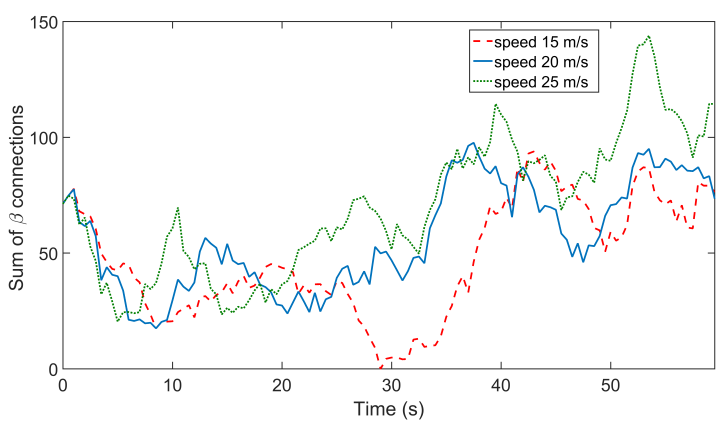

Figure 16: Effect of changing speed on the performance of the mGMC. 

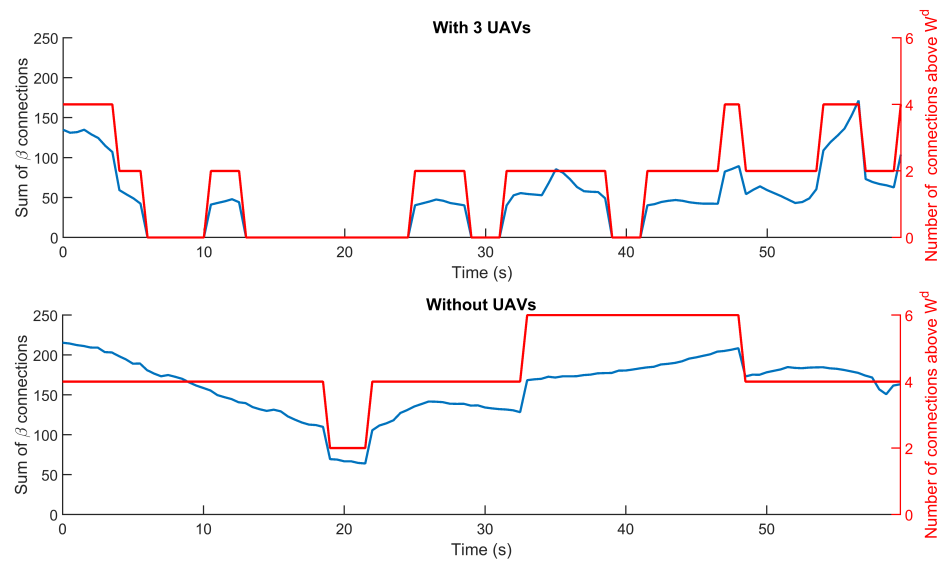

Figure 17: Comparison of performance $\left(J_{m G M C}\right.$ cost $)$ between cases with and without UAVs.

weak connections to improve than if a small number of UAVs is present (e.g. it is possible a single connection is improved by two UAVs in a chain rather than a single UAV).

Different heading rates and speeds are used to determine how UAV dynamics affects the communication performance. Figures 15 and 16 show that higher turning rates and slower speeds results in better communication performance in terms of lowering the communication cost. In these conditions, UAVs can make tighter turns, thus they can better react to the local changes of ground node positions and corresponding communication structure (i.e. MST).

To illustrate a significant reduction of the number of weak connections using the proposed algorithm, the mGMC cost and the number of the weak connection (communication quality above $W^{d}$ ) of a sample scenario with and without using UAVs is shown in Fig.17. In this scenario, UAVs cause a significant reduction of number of weak connections where the average number of weak connections with UAVs is about 1.6, while without the UAV is 4.4, proving the benefit of the proposed approach.

Currently, control commands need to be sent to UAVs every half a second, so each NMPC iteration needs to be finished within that time. It is also worth noting that algorithm can be run in a decentralized manner (i.e. each UAV performs its own NMPC computation based on the prediction on what the rest of the group will do). Thus, computation time per UAV is representative of how fast the group can finish one iteration. Table 3 shows the computation cost of one iteration, implemented in Matlab, using the same scenario described before with and without buildings in the environment. This implies the significant impact of LOS obstruction detection on the computation time. Figure 11 represents the computation time with respect to the number of ground vehicle. Possible approaches to address the issue on the computation time include: i) implement the algorithm in $\mathrm{C} / \mathrm{C}++$. Using a lower level language will reduce computation time significantly; ii) commands can be sent less often to the UAV. This will increase the allowed 
time for each iteration to finish at the expense of communication performance of the algorithm; and iii) the number of iterations or population size of the genetic algorithm can be reduced to achieve significant reduction of the computation time. However, this is likely to reduce the quality of solution.

Table 4: Mean and standard deviation for a runtime per iteration averaged over ten sample scenarios

\begin{tabular}{ccc}
\hline Computation time & Without buildings & With buildings \\
\hline \hline Mean time \pm Std $(\mathrm{sec})$ & $0.29 \pm 0.0088$ & $8.9 \pm 0.55$ \\
\hline
\end{tabular}

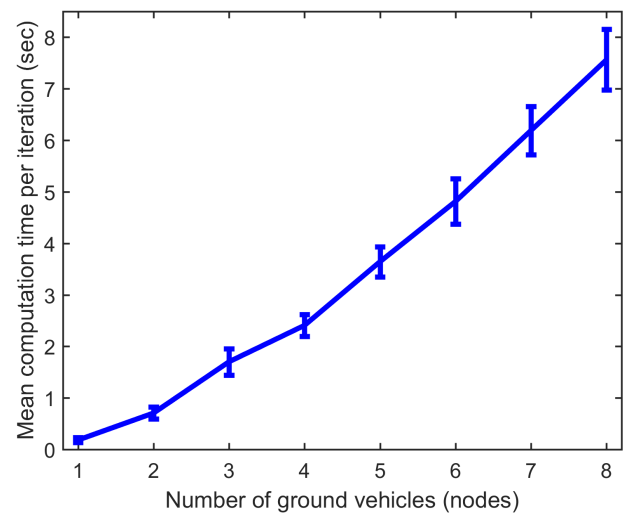

Figure 18: Computation time for a single UAV and 30 buildings with respect to the number of ground vehicles.

\section{Conclusions and Future Work}

This paper has presented a trajectory planning algorithm for fixed-wing communication relay UAVs to enhance communication quality of the ground mobile network in an urban environment. To plan the optimal trajectory, discretised NMPC trajectory planning was proposed using a genetic algorithm-based optimisation method. The planner was paired with the mGMC communication performance metric targeted to improve only the necessary weak connections in the network. How different horizon length, the number of the UAV used, and kinematic constraints of the UAV such as the heading rate and the speed can affect the communication performance was shown and discussed along with benefits of using the proposed algorithm over a scenario without UAVs was shown and discussed. Future work will include more accurate and realistic communication model and decision making regarding the necessary number of the UAVs in the mission. 


\section{References}

1. D.G. Press. Urban warfare: Options, problems and the future, Janaury 1999.

2. A.S. Ibrahim, K.G. Seddik, and K.J.R. Liu. Improving connectivity via relays deployment in wireless sensor networks. GLOBECOM - IEEE Global Telecommunications Conference, pages 1159-1163, 2007.

3. E.P. De Freitas, T. Heimfarth, I.F Netto, C. Eduardo Lino, C.E. Pereira, A.M. Ferreira, F.R Wagner, and T. Larsson. UAV relay network to support WSN connectivity. International Congress on Ultra Modern Telecommunications and Control Systems and Workshops, pages 309-314, 2010.

4. S. Gil, M. Schwager, B.J. Julian, and D. Rus. Optimizing communication in air-ground robot networks using decentralized control. IEEE International Conference on Robotics and Automation, pages 1964-1971, 2010.

5. C. Dixon and E.W. Frew. Optimizing cascaded chains of unmanned aircraft acting as communication relays. IEEE Journal on Selected Areas in Communications, 30(5):883$898,2012$.

6. D.-T. Ho, E.I. Grotli, P.B. Sujit, T.A. Johansen, and J.B. De Sousa. Performance evaluation of cooperative relay and Particle Swarm Optimization path planning for UAV and wireless sensor network. IEEE Globecom Workshops, pages 1403-1408, 2013.

7. A.J. Carfang, N. Wagle, and E.W. Frew. Improving data ferrying by iteratively learning the radio frequency environment. IEEE International Conference on Intelligent Robots and Systems, (Iros):1182-1188, 2014.

8. P. Basu, J. Redi, and V. Shurbanov. Coordinated flocking of UAVs for improved connectivity of mobile ground nodes. IEEE Military Communications Conference, 3:1628-1634, 2004.

9. S. Kim, H. Oh, J. Suk, and A. Tsourdos. Coordinated trajectory planning for efficient communication relay using multiple UAVs. Control Engineering Practice, 29:42-49, 2014.

10. Z. Han, L. Swindlehurst, and K.J.R. Liu. Optimization of MANET Connectivity Via Smart Deployment / Movement of Unmanned Air Vehicles. IEEE Transactions on Vehicular Technology, 58(7):3533-3546, 2009.

11. S. Kim, P. Silson, A. Tsourdos, and M. Shanmugavel. Dubins path planning of multiple unmanned airborne vehicles for communication relay. Proceedings of the Institution of Mechanical Engineers, Part G: Journal of Aerospace Engineering, 225(1):12-25, 2011.

12. D.H. Choi, B.H. Jung, and D.K. Sung. Low-complexity Maneuvering Control of a UAVbased Relay without Location Information of Mobile Ground Nodes. IEEE Symposium on Computers and Communication, pages 1-6, 2014.

13. H. Oh, H.S. Shin, S. Kim, P. Ladosz, and W.H. Chen. Communication-aware convoy following guidance for UAVs in a complex urban environment. In The 24th Mediterranean Conference on Control and Automation, Athens, Greece, 2016.

14. P. Ladosz, H. Oh, and W.-H. Chen. Optimal positioning of communication relay unmanned aerial vehicles in urban environments. In The 2016 International Conference on Unmanned Aircraft Systems, Washington, USA, 2016.

15. D. Legland. geom3d, June 2009. http://www.mathworks.com/matlabcentral/fileexchange/24484geom3d.

16. R. Sedgewick and K. Wayne. Minimum spanning trees, 2015. http://algs4.cs.princeton.edu/43mst/.

17. J. Kennedy and R. Eberhart. Particle swarm optimization. IEEE International Conference on Neural Networks, 4:1942-1948 vol.4, 1995.

18. S. Kim, H. Oh, and A. Tsourdos. Nonlinear model predictive coordinated standoff tracking of a moving ground vehicle. Journal of Guidance Control and Dynamics, 36:557-566, August 2013.

19. K. Mehrotra and P. R. Mahapatra. A jerk model for tracking highly maneuvering targets. IEEE Transactions on Aerospace and Electronic Systems, 33(4):1094-1105, 1997.

20. V. Shaferman and T. Shima. Unmanned Aerial Vehicles Cooperative Tracking of Moving Ground Target in Urban Environments. Journal of Guidance, Control, and Dynamics, 31(5):1360-1371, 2008.

21. J. Chinneck. Practical optimization: A gentle introduction, 2015. http://www.sce.carleton.ca/faculty/chinneck/po.html. 